\section{The effect of frequency on the auditory evoked response}

THOMAS R. EVANS and BRUCE $H$. DEATHERAGE, Sensory Information Research Laboratory, TRACOR, Inc., Austin, Texas 78721

This study investigated the effects of frequency on the amplitude of the cortical evoked response as recorded from the cranial vertex. The stimuli were monaurally presented tones, $200 \mathrm{msec}$ in duration, with frequencies of $500,1000,2000$, or $4000 \mathrm{~Hz}$. Stimulus intensities of 90-dB hearing level (HL) and 45-dB hearing level (HL) were examined. A negative relationship was found between the amplitude of the evoked response and the frequency of the stimulus. At both intensity levels, the amplitude of the response decreased progressively as the frequency increased.

The effects of various parameters of acoustic stimulation on the amplitude of the auditory evoked response have been examined by a number of investigators. Stimulus intensity (Davis \& Zerlin, 1966; Davis, Bowers, \& Hirsh, 1968; Butler, 1968), duration and rise time (Onishi \& Davis, 1968 ), binaural summation (Davis \& Zerlin, 1966), and masking, both tonal (Butler, 1968) and broad-band (Davis, Bowers, \& Hirsh, 1968) have all been shown to have an effect on the amplitude of the evoked response as measured from the $N_{1}$ peak to the $\mathrm{P}_{2}$ "trough." The effect of frequency on this cortical response, however, appears somewhat ambiguous. Davis \& Zerlin (1966) were unable to find any significant relation between frequency and the amplitude of the response at the vertex. In a later study, however, Davis et al (1968), in examining the relations of the evoked response to loudness and masking, observed that the exponent of the power function relating the amplitude of the response to signal intensity was different for the three frequencies used. The differences observed were in the direction of a decreasing exponent for increasing frequency. Somewhat indirectly related is the observation by Butler (1968) of an effect of frequency interaction on the evoked response. The study reported here was designed specifically to investigate the relationship of frequency to the amplitude of the auditory evoked response.

\section{METHOD}

The Os were four young men (ages 18-23) with clinically-normal hearing who were presented with tone bursts generated by a Rudmose RA 114 Audiometer. The signals had frequencies of $500,1000,2000$, and
$4000 \mathrm{~Hz}$. Signal intensities of 90 - and $45-\mathrm{dB}$ hearing level (HL) calibrated in accordance with ISO-1963 standards were used. All signals had a duration of $200 \mathrm{msec}$ with a 10 -msec rise-decay time. Signals were presented monaurally through headphones while the Os were seated in soundproof rooms.

Bipolar recordings were taken with one active electrode located on the vertex of the skull, a second active electrode located on the forehead, and a third, reference electrode located on the mastoid area of the skull.

Responses were averaged over 80 stimulus presentations by a Northem Scientific Digital Memory Oscilloscope and were photographed with a Polaroid camera. Fifteen such averages were taken at each signal frequency and each intensity level for each 0 .

The amplitude of the evoked response was measured from the $\mathrm{N}_{1}$ (negative) peak to the $P_{2}$ (positive) peak by means of a grid tensity (re: $500-\mathrm{Hz}$ response). disided into $.25-\mathrm{mm}$ units. The amplitudes were evaluated as differences relative to the $500 \mathrm{Il}$ response for each 0 . These purcentage values were then averaged over the four Os.

\section{RESULTS}

The results are summarized in Fig. 1. Figure 1 shows the relative amplitudes of the evoked responses, referenced to the response amplitude associated with $500 \mathrm{~Hz}$, as a function of frequency and intensity. However, when parametrically assessing the effects of an auditory stimulus, it may be more meaningful to express the intensity of the stimulus in unmodified physical units. Since hearing level (HL) is an audiometric zero reference level, equal hearing level intensities may not be equal in terms of sound pressure level. The levels used in this study, therefore, are translated along the left-hand ordinate of Fig. 1 into their equivalent sound pressure levels (decibels re $0.0002 \mathrm{dynes} / \mathrm{cm}^{2}$ ) at the frequencies used. The percentage difference in the amplitude of the evoked responses is indicated along the right-hand ordinate.

As shown in Fig. 1, at $90 \mathrm{~dB}$ HL there is a progressive decrease in the relative amplitude of the evoked response as the frequency increases (linear trend:

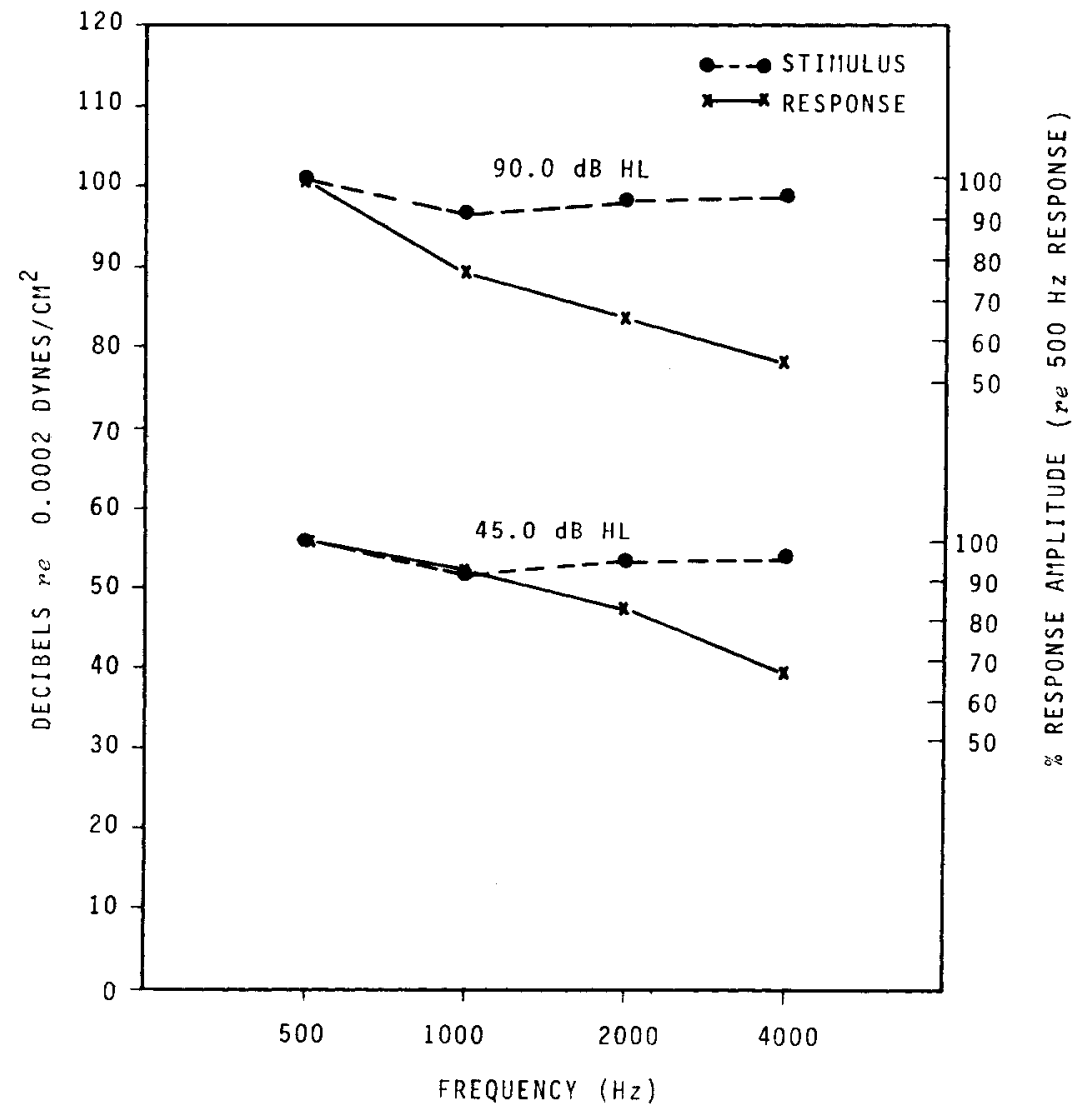

Fig. 1. Relative evoked response amplitude as a function of frequency and in- 
$\mathrm{F}=76.40, \mathrm{p}<.01)$. The $4000-\mathrm{Hz}$ response has an amplitude $45 \%$ smaller than that observed in response to the $500-\mathrm{Hz}$ tone with similar but proportionately smaller differences observed at the other frequencies. At $45 \mathrm{~dB}$ HL, a similar decrease in amplitude as a function of frequency (linear trend: $F=61.78, p<.01)$ is observed. Although the decrease in amplitude observed at $1000 \mathrm{~Hz}(-07 \%)$ can be accounted for by the difference in the sound pressure levels of the $500-$ and $1000-\mathrm{Hz}$ tones, a progressive decrease is evidenced at the other frequencies which cannot be so interpreted.

\section{DISCUSSION}

The observation that, for essentially equal intensity conditions, the amplitude of the evoked response decreases with increases in frequency requires a speculative explanation. The results of earlier studies dealing with evoked responses suggest that the amplitude of the response is more linearly related to the subjective dimension of loudness than to the objective dimensions of frequency and intensity. Such a relationship would anticipate an effect of frequency manifest as changes in amplitude which are correlated with the auditory thresholds at those frequencies. Consequently, the loudness hypothesis would predict that, at equal intensities, the amplitude of the response would be greatest between 1000 and 2000 $\mathrm{Hz}$ and progressively smaller on either side of this frequency range. The limited frequency range as well as the high signal intensities used in this study preclude any definitive conclusion concerning an effect related to loudness. However, the progressive decrease in amplitude observed suggests that an alternative explanation is required.

One such explanation is that the amplitude of the evoked response is related to the number of neural elements activated at the cochlea. Since the $500-\mathrm{Hz}$ tone produces a disturbance over a larger area of the basilar membrane than the 1000-, 2000-, or $4000-\mathrm{Hz}$ tones, the $1000-\mathrm{Hz}$ tone produces a disturbance over a larger area than the $2000-$ or $4000-\mathrm{Hz}$ tones, etc., the concomitant number of neural elements activated by each frequency would decrease as the frequency increased-a relationship consistent with that observed between frequency and evoked response amplitude. Although the explanation is appealing from a parsimonious point of view, more detailed investigation is required for a more conclusive statement.

\section{REIERENCES}

BUTLER, R. A. Effects of changes in stimulus frequency and intensity on habituation of the human vertex potential. Journal of the Acoustical Society of America, 1968, 44, $945-950$.

DAVIS, H.. \& ZERLIN, S. Acoustic relations of the human vertex potential. Joumal of the Acoustical Society of America, 1966, 39 , $109-116$.

DAVIS, H., BOWERS, C., \& HIRSH, S. K. Relations of the human vertex potential to acoustic input: Loudness and masking. Joumal of the Acoustical Society of America, 1968,43, $431-438$.

ONISHI, S., \& DAVIS, H. Effects of duration and rise time of tone bursts on evoked $V$ potentials. Journal of the Acoustical Society of America, $1968,44,582-591$.

\section{Retention and utilization of mediators by retardates'}

JOHN G. BORKOWSKI, JOSEPH P. AHEARN, ${ }^{2}$ and JOHN PEARSON, University of Notre Dame, Notre Dame, Ind. 46556

The purpose of this study was to assess the ability of mildly retarded children to store and utilize mediational links in the $A-B, B-C, A-C$ paradigm. For three chaining groups the intervals between Lists $B-C$ and $A \cdot C$ were $30 \mathrm{sec}, 20 \mathrm{~min}$, or $24 \mathrm{~h}$. The interval between $D-C$ and $A-C$ in a control group $(A-B, D-C, A-C)$ was $30 \mathrm{sec}$. The major results showed that the $A-C$ learming for the control group was inferior to that for the mediation groups which were not different from one another.

A number of studies have demonstrated the ability of mentally retarded individuals to utilize mediational association during paired-associate (PA) learning in the chaining paradigm (Berkson \& Cantor, 1960; Borkowski \& Johnson, 1968). In this paradigm (i.e., Lists A-B, B-C, A-C), the occurrence of $A$ in Stage 3 has a tendency to elicit $B$ and the occurrence of $B$ in turn tends to evoke $C$, the correct response to the $A$ stimulus. Although there is evidence for the facilitating effect of mediation on A-C acquisition, there are limited data dealing with the ability of retardates to retain mediational links. Jensen \& Rohwer (1963) found that when retardates were presented sentences which related the stimulus and response terms of a PA list, relearning of the material after an interval of 1 week was not significantly different from the relearning of a control group having no prior "sentencemediation" during acquisition. However, a high degree of original learning for the two groups, toge ther with a relearning measure of retention, prevented an unequivocal interpretation of these results.

The present study attempted to assess more directly the ability of retardates to store and utilize highly available mediating links. Specifically, three groups of retarded Ss learned an A-C list $30 \mathrm{sec}, 20 \mathrm{~min}$, or $24 \mathrm{~h}$ after learning Lists A-B and B-C. A single control group learned List A-C $30 \mathrm{sec}$ after learning List $D-C$. Since the materials were common pictures and the criteria for the learning of Lists A-B and B-C were extremely high, it was hypothesized that the retention in terval would have minimal effect on the retardates' utilization of mediators during A-C learning.

\section{SUBJECTS}

The Ss were 78 institutionalized, mentally-re tarded children (mean IQ of 49.1 and mean $\mathrm{CA}$ of 16.2 years) from Fort Wayne State Hospital and Training Center. The majority of Ss showed some form of neurological damage. Seventeen per cent of the sample was female and $95 \%$ white. Seven Ss were unable to follow instructions or failed to reach criterion in five trials during the learning of List 1 or 2

In general, Ss were randomly assigned to the four conditions. However, since the population was limited in size, fewer patients were assigned to the control condition $(\mathrm{N}=16)$ than to the mediation conditions (Ns of 18, 18, and 19 for the $30-\mathrm{sec}, 20 \mathrm{~min}$, and $24 \mathrm{~h}$ groups, respec. tively). Ss were assigned to conditions so as to equate for mean ages and IQs.

\section{MATERIALS}

The materials and procedure were essentially similar to those used by Borkowski \& Johnson (1968). The specific materials used in the chaining (A-B, B-C, $A-C)$ and control (A-B, D-C, A-C) paradigms were highly associated object-pictures as the $A$ and $B$ terms and colors as the $C$ terms. The $C$ terms were highly associated with the B stimuli but not with the A stimuli. An example of one set of pairs used in the mediational list is: boy (A)-wagon (B), wagon (B)-red (C), boy (A)-red (C). The control condition had pictures as $D$ terms which were associated with C (e.g., apple-red) but not with $A$ or B. Six pairs 